\title{
Absolute intensity calibration of the Wendelstein 7-X high efficiency extreme ultraviolet overview spectrometer system
}

Albert Greiche, Wolfgang Biel, Oleksandr Marchuk, and Rainer Burhenn

Citation: Review of Scientific Instruments 79, 093504 (2008);

View online: https://doi.org/10.1063/1.2977541

View Table of Contents: http://aip.scitation.org/toc/rsi/79/9

Published by the American Institute of Physics

\section{Articles you may be interested in}

Layout and results from the initial operation of the high-resolution x-ray imaging crystal spectrometer on the Large Helical Device

Review of Scientific Instruments 83, 083506 (2012); 10.1063/1.4744935

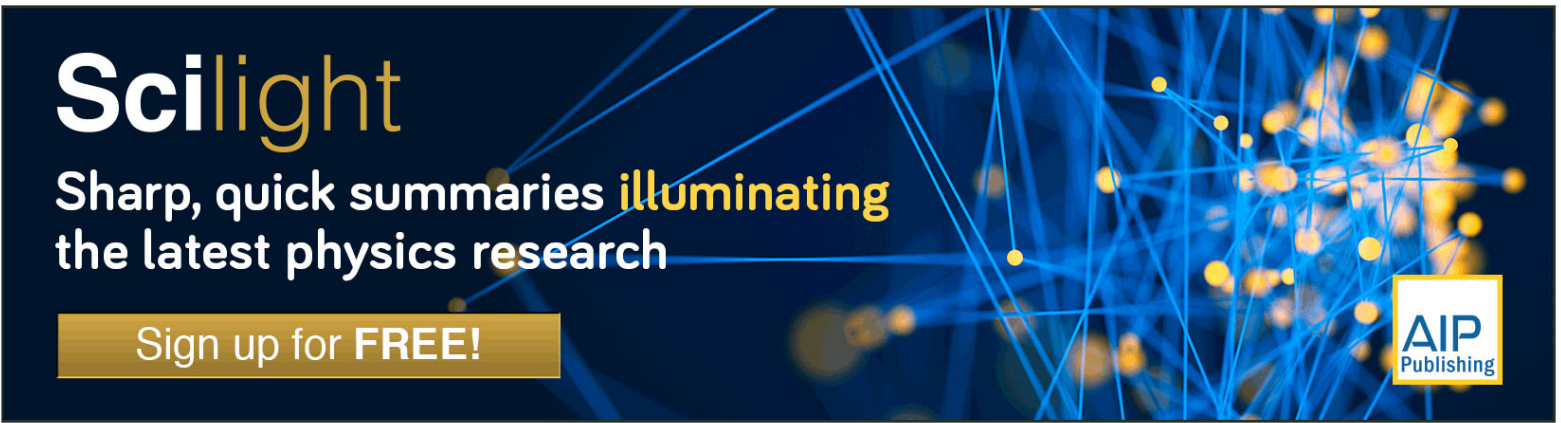




\title{
Absolute intensity calibration of the Wendelstein 7-X high efficiency extreme ultraviolet overview spectrometer system
}

\author{
Albert Greiche, ${ }^{1, a)}$ Wolfgang Biel, ${ }^{1}$ Oleksandr Marchuk, ${ }^{1}$ and Rainer Burhenn ${ }^{2}$ \\ ${ }^{1}$ Institut für Energieforschung-Plasmaphysik, Forschungszentrum Jülich GmbH, EURATOM Association, \\ Trilateral Euregio Cluster, D-52425 Jülich, Germany \\ ${ }^{2}$ Max-Planck-Institut für Plasmaphysik, EURATOM Association, D-17491 Greifswald, Germany
}

(Received 14 March 2008; accepted 11 August 2008; published online 9 September 2008)

The new high effiency extreme ultraviolet overview spectrometer (HEXOS) system for the stellarator Wendelstein 7-X is now mounted for testing and adjustment at the tokamak experiment for technology oriented research (TEXTOR). One part of the testing phase was the intensity calibration of the two double spectrometers which in total cover a spectral range from 2.5 to 160.0 $\mathrm{nm}$ with overlap. This work presents the current intensity calibration curves for HEXOS and describes the method of calibration. The calibration was implemented with calibrated lines of a hollow cathode light source and the branching ratio technique. The hollow cathode light source provides calibrated lines from 16 up to $147 \mathrm{~nm}$. We could extend the calibrated region in the spectrometers down to $2.8 \mathrm{~nm}$ by using the branching line pairs emitted by an uncalibrated pinch extreme ultraviolet light source as well as emission lines from boron and carbon in TEXTOR plasmas. In total HEXOS is calibrated from 2.8 up to $147 \mathrm{~nm}$, which covers most of the observable wavelength region. The approximate density of carbon in the range of the minor radius from 18 to $35 \mathrm{~cm}$ in a TEXTOR plasma determined by simulating calibrated vacuum ultraviolet emission lines with a transport code was $5.5 \times 10^{17} \mathrm{~m}^{-3}$ which corresponds to a local carbon concentration of 2\%. () 2008 American Institute of Physics. [DOI: 10.1063/1.2977541]

\section{INTRODUCTION}

In fusion experiments the properties of magnetically confined hydrogen plasmas strongly depend on the amount of impurities and their transport parameters. Impurities are either produced by the fusion process $(\mathrm{He})$ or by plasma-wall contact (Be, B, C, N, O, Si, W, metals). They also can be added on purpose to either modify the plasma properties or to analyze the transport properties (e.g., $\mathrm{He}, \mathrm{N}, \mathrm{Ne}, \mathrm{Ar}, \mathrm{Ti}$ ).

For impurity monitoring and impurity transport experiments on the stellarator Wendelstein 7-X (W7-X) the new high effiency extreme ultraviolet (XUV) overview spectrometer (HEXOS) system has been developed. ${ }^{1}$ During the construction phase of W7-X, HEXOS was mounted at the tokamak experiment for technology oriented research (TEXTOR) for instrumental tests and adjustments. The adjustments include an absolute intensity calibration of the spectrometer system ${ }^{2,3}$ by means of a calibrated hollow cathode light source which provides absolutely calibrated lines from 16 up to $147 \mathrm{~nm} .{ }^{4,5}$ The wavelength region inaccessible by the hollow cathode light source was calibrated with branching line pairs of a pinched laboratory XUV source (AIXUV GmbH) ${ }^{6}$ and boron and carbon lines of TEXTOR plasmas.

In conjunction with equilibrium calculations of a transport code, it is possible to estimate the local impurity densities and therefore the local impurity concentrations in the fusion plasmas of TEXTOR.

In Sec. II we describe the HEXOS system, in Secs. III and IV the calibration procedure, and in Sec. V an example

${ }^{a)}$ Electronic mail: a.greiche@fz-juelich.de. for the application of the HEXOS calibration to TEXTOR plasmas. Section VI summarizes all results.

\section{HEXOS SETUP}

The spectrometer system HEXOS consists of two double spectrometers which cover a spectral range from 2.5 up to $160 \mathrm{~nm}$ (Table I). In these spectral regions all relevant impurities which occur in magnetically confined plasmas can be observed. As described in Ref. 1 HEXOS uses newly developed toroidal holographic diffraction gratings with up to 2000 ion etched grooves per millimeter. These gratings are the only optical and dispersive elements in the system. The diffraction gratings image the entrance slit on the detectors which are localized in the focal plane of the gratings. The detectors consist of an open microchannel plate (MCP) from Burle Industries Inc. with CsI coating and extended dynamic range (channel length $L /$ diameter $D=60: 1$, pore size $=10 \mu \mathrm{m}$, diameter $=40 \mathrm{~mm}$ ) which detects and amplifies the signal from the photons and a P47-phosphor screen onto which the secondary electrons convert the electrical signal into visible light. The screen and MCP are supplied with a high voltage so that this device has to be operated in ultrahigh vacuum conditions to avoid electrical discharges which can cause damage to the MCP.

In order to achieve the required vacuum inside the spectrometer chamber in the presence of the open slit at the entrance, the beamline between the entrance slit and TEXTOR is additionally pumped (differential pumping). The spectra on the phosphor screen are recorded by a camera ${ }^{7}$ with a linear 1024 pixel array and a time resolution of $1 \mathrm{~ms}$, which 
TABLE I. The spectral range, mean etendue, and operating width of the entrance slits at TEXTOR of each HEXOS spectrometer.

\begin{tabular}{|c|c|c|c|c|}
\hline Spectrometer No. & 1 & 2 & 3 & 4 \\
\hline Spectral range $(\mathrm{nm})$ & $2.5-10.5$ & $9-24$ & $20-66$ & $60-160$ \\
\hline Average etendue $\left(10^{-4} \mathrm{~mm}^{2} \mathrm{sr}\right)$ & 0.30 & 1.02 & 2.07 & 2.10 \\
\hline Entrance slit $(\mu \mathrm{m})$ & 220 & 120 & 60 & 60 \\
\hline
\end{tabular}

is the typical time scale for impurity transport processes in a fusion plasma.

For the calibration during the laboratory testing phase, the width of each entrance slit of HEXOS was set to $30 \mu \mathrm{m} .{ }^{8}$ The widths of the entrance slits were changed before mounting them at TEXTOR to achieve the optimized etendues (see Table I).

\section{CALIBRATION OF SPECTROMETERS 2-4}

HEXOS was calibrated during the laboratory testing phase. ${ }^{8}$ As secondary standard calibration light source we used a high current hollow cathode which was absolutely calibrated with synchroton radiation at the Berliner Elektronenspeicherring-Gesellschaft für Synchrotronstrahlung m.b.H. (BESSY). Table II shows the calibrated lines of the hollow cathode at the working points for $I_{\mathrm{HC}}$ of 1 (Refs. 4 and 5) and $2 \mathrm{~A}$ (Ref. 9) and additional data that were used during the calibration process.

To check the grating efficiency in the case of a spatially extended fusion plasma and fully illuminated grating surface, respectively, the pointlike calibration source was moved in the plane perpendicular to the optical axis. The grating efficiency did not depend on the position of the light source. Spectrometers 3 and 4 were both calibrated with the tabulated lines emitted by the hollow cathode (Fig. 1). The calibration of each spectrometer was performed with a detector voltage of $1150 \mathrm{~V}$ and a phosphor screen voltage of $7500 \mathrm{~V}$. For spectrometer 2 the hollow cathode provided calibrated lines down to $16 \mathrm{~nm}$ (Fig. 2). The shorter wavelengths can only be calibrated by branching line pairs because there is no calibrated light source available for this wavelength region.

For the calibration the power output $P$ of each emission line of the hollow cathode which passed the entrance slit of the spectrometer was compared to the measured detector signal $S$ integrated over the line width. $P$ was calculated via the solid angle determined by the distance to the entrance slit with respect to the light source,

$$
x=\frac{P}{S} .
$$

With $x$ we calculated the number of photons that passed the slit during the time interval of the measurement. With the help of the mean etendue $L_{m}$ (see Table I) of each spectrometer we determined the inverse sensitivity $p^{-1}$ for each line as follows:

$$
p^{-1}=\frac{x \lambda}{h c L_{m}},
$$

in which $h \cdot c / \lambda$ is the energy of one photon of the observed line.

\begin{tabular}{|c|c|c|c|c|c|c|}
\hline $\begin{array}{l}\text { Spectrometer } \\
\text { No. }\end{array}$ & Ion & $\begin{array}{c}\lambda \\
(\mathrm{nm})\end{array}$ & $\begin{array}{l}I_{\mathrm{HC}} \\
(\mathrm{A})\end{array}$ & $\begin{array}{c}P \\
(\mathrm{pW})\end{array}$ & $\begin{array}{c}S \\
\text { (counts/ms) }\end{array}$ & $\begin{array}{c}p^{-1} \\
{\left[\text { photons } /\left(\text { counts } \mathrm{cm}^{2} \mathrm{sr}\right)\right]}\end{array}$ \\
\hline 2 & $\mathrm{Al} \mathrm{IV}, \mathrm{He}$ & 16.10 & 2 & 0.18 & 1 & $1.54 \times 10^{7}$ \\
\hline 2 & He II & 24.30 & 2 & 5.50 & 31 & $2.10 \times 10^{7}$ \\
\hline 3 & He II & 24.30 & 2 & 5.50 & 12 & $2.63 \times 10^{7}$ \\
\hline 3 & He II & 25.60 & 2 & 17.39 & 49 & $2.19 \times 10^{7}$ \\
\hline 3 & He II & 30.40 & 2 & 79.96 & 242 & $2.44 \times 10^{7}$ \\
\hline 3 & $\mathrm{Ne}$ II & 40.65 & 1 & 17.63 & 49 & $3.55 \times 10^{7}$ \\
\hline 3 & $\mathrm{Ne} I I$ & 46.10 & 1 & 121.25 & 339 & $4.01 \times 10^{7}$ \\
\hline 3 & $\mathrm{Ne}$ III & 49.00 & 1 & 11.71 & 33 & $4.25 \times 10^{7}$ \\
\hline 3 & Ar II & 54.30 & 1 & 3.35 & 9 & $4.95 \times 10^{7}$ \\
\hline 3 & Ar II & 54.75 & 1 & 3.89 & 10 & $5.18 \times 10^{7}$ \\
\hline 3 & He I & 58.40 & 2 & 180.98 & 486 & $5.29 \times 10^{7}$ \\
\hline 3 & Ar II & 61.24 & 1 & 0.89 & 2 & $5.56 \times 10^{7}$ \\
\hline 4 & $\mathrm{Ne} \mathrm{I}$ & 73.59 & 1 & 68.25 & 528 & $2.28 \times 10^{7}$ \\
\hline 4 & $\mathrm{Ne} \mathrm{I}$ & 74.37 & 1 & 45.07 & 391 & $2.05 \times 10^{7}$ \\
\hline 4 & Ar III & 76.92 & 1 & 1.85 & 17 & $2.06 \times 10^{7}$ \\
\hline 4 & $\mathrm{Kr}$ II & 88.63 & 1 & 11.46 & 79 & $3.09 \times 10^{7}$ \\
\hline 4 & $\mathrm{Kr}$ II & 91.74 & 1 & 21.53 & 160 & $2.95 \times 10^{7}$ \\
\hline 4 & Ar II & 91.98 & 1 & 45.90 & 372 & $2.72 \times 10^{7}$ \\
\hline 4 & Ar II & 93.21 & 1 & 28.15 & 223 & $2.82 \times 10^{7}$ \\
\hline 4 & $\mathrm{Kr}$ II & 96.50 & 1 & 23.78 & 234 & $2.34 \times 10^{7}$ \\
\hline 4 & Ar I & 104.82 & 1 & 24.96 & 286 & $2.19 \times 10^{7}$ \\
\hline 4 & Ar I & 106.62 & 1 & 20.82 & 281 & $1.89 \times 10^{7}$ \\
\hline 4 & $\mathrm{Kr} \mathrm{I}$ & 116.49 & 1 & 5.86 & 72 & $2.26 \times 10^{7}$ \\
\hline 4 & $\mathrm{Kr} \mathrm{I}$ & 123.58 & 1 & 16.09 & 184 & $2.59 \times 10^{7}$ \\
\hline 4 & Xe I & 146.96 & 1 & 9.79 & 89 & $3.89 \times 10^{7}$ \\
\hline
\end{tabular}

TABLE II. Spectral lines and additional data used for the calibration of HEXOS (Refs. 4, 5, and 9). 


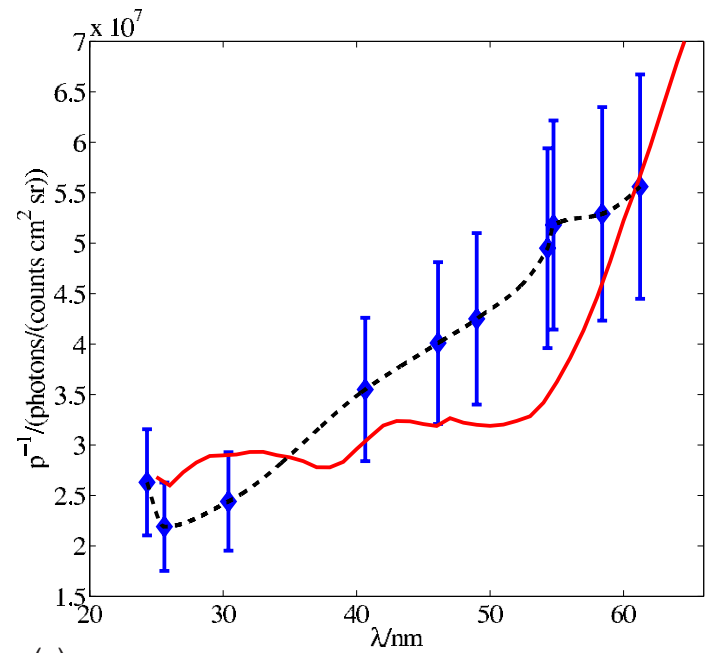

(a)

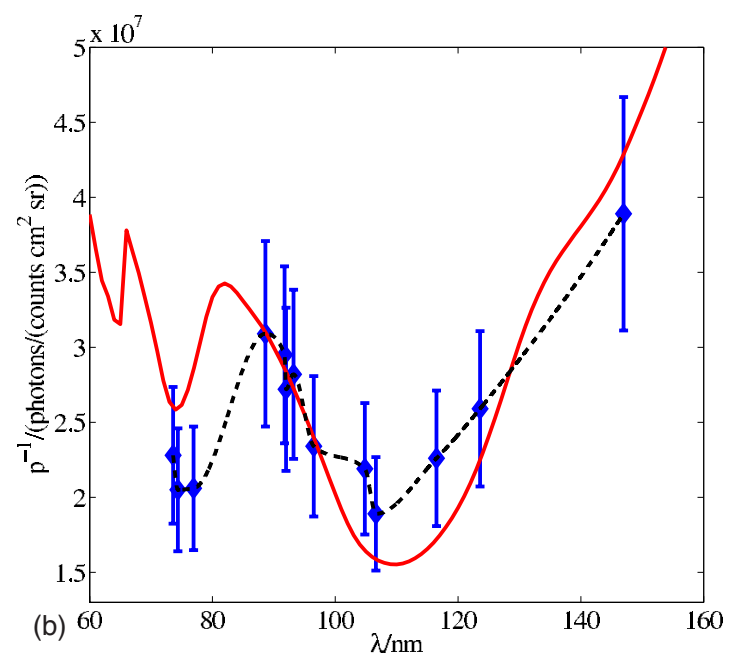

FIG. 1. (Color online) Inverse sensitivities for (a) spectrometer 3 and (b) spectrometer 4 . The diamonds are the data points obtained with the hollow cathode being fitted by a spline (dashed curve). The deviations between the calculated and the measured data arise due to differences in the compared MCPs.

We estimated an expected inverse sensitivity by multiplying the angle dependent MCP quantum efficiency based on Refs. 10 and 11 with the grating efficiency which was obtained by the software PCGRATE from International Intellectual Group, Inc. and a correction factor for the detector voltages derived by laboratory measurements. Figure 1 shows the calibration curves for spectrometers 3 and 4, respectively. The diamonds are the values derived by the hollow cathode measurements and the continuous line represents the expected inverse sensitivity. Taking into account an error of $\pm 18 \%$ to $20 \%$ (calculated from the root of the square sum of the accuracy of the hollow cathode voltage $(5 \%)$, the long term stability of the hollow cathode $(10 \%)$, the accuracy of the line integration (10\%), and the error of the tabulated calibration lines (10\%-13\%) and considering the differences between the MCP parameters of Refs. 7, 10, and 11 the measured data fit well to the calculated curve. To obtain a continuous calibration curve for the whole spectral range of each spectrometer, the experimental data were fitted by splines (dashed curves).

The maximum in the inverse sensitivity around $90 \mathrm{~nm}$ in

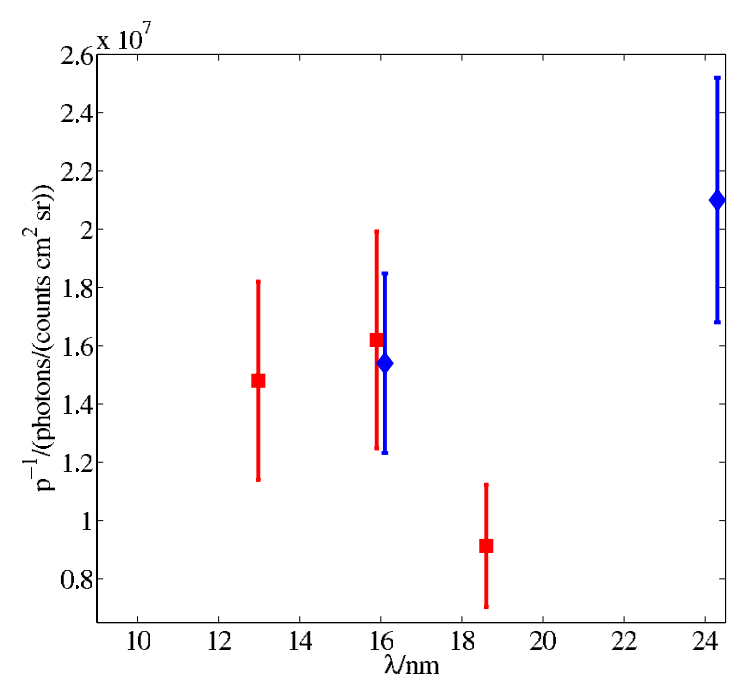

FIG. 2. (Color online) Inverse sensitivity of spectrometer 2. The diamonds are the data points obtained with the hollow cathode and the squares are the data points obtained with the branching ratio technique using the laboratory pinch light source.

Fig. 1(b) results from the effect that around this photon energy it is possible to excite more than one photoelectron in CsI. Since these two electrons have a lower escaping probability due to their lower kinetic energy, the photoelectron yield becomes less effective. ${ }^{11}$ With a rising photon energy, the yield rises again and the inverse sensitivity drops.

\section{CALIBRATION OF SPECTROMETERS 1 AND 2}

There are no secondary standard light sources in the spectral range of spectrometer 1 and in parts of spectrometer 2. Therefore, an absolute intensity calibration using the branching ratio technique has to be applied, which utilizes the known intensity ratio (branching ratio) of line pairs being emitted during transitions from the same upper excitation level. ${ }^{12-14}$ Usable line pairs for this method which are typically emitted by our pinch light source ${ }^{6}$ as well as from TEXTOR plasmas are listed in Table III.

In order to successfully apply this method for the calibration of the wavelength region below $16 \mathrm{~nm}$, well separated, optically thin line pairs are needed, with one line located in the spectral range to be calibrated and the other line emitted in the calibrated wavelength range. If the transition probabilities for the chosen lines are known ${ }^{12,15-18}$ the intensity $I_{s}$ at the uncalibrated shorter wavelength $\lambda_{s}$ can be calculated with the help of the intensity $I_{l}$ of the line in the calibrated spectral range at the longer wavelength $\lambda_{l}$ as

$$
I_{s}=\frac{A_{s} \lambda_{l}}{A_{l} \lambda_{s}} I_{l},
$$

with $A_{s}$ and $A_{l}$ being the corresponding transition probabilities. $^{12}$

For spectrometer 2 the hollow cathode provides two usable calibration points, $\mathrm{Al}$ IV $16 \mathrm{~nm}$ in the helium sputtering regime and $\mathrm{He}$ II $24.3 \mathrm{~nm}$. Additional calibration points with branching line pairs were obtained in the laboratory testing phase with the pinch light source from AIXUV GmbH: O VI 12.9/49.8 nm, Ar VIII 15.9/33.7 nm, and N V 18.6/71.4 nm. 
TABLE III. Transition probabilities of the line pairs used for the branching ratio technique (Refs. 12 and 15-18). Spectrometer 1 was calibrated with boron and carbon from TEXTOR plasmas and spectrometer 2 with the pinch light source (oxygen, argon, and nitrogen).

\begin{tabular}{|c|c|c|c|c|c|c|c|c|c|}
\hline Ion & $\begin{array}{c}\lambda_{s} \\
(\mathrm{~nm})\end{array}$ & $\begin{array}{c}A_{s} \\
\left(\mathrm{~s}^{-1}\right)\end{array}$ & $\begin{array}{c}S_{s} \\
\text { (counts } / \mathrm{ms})\end{array}$ & $\begin{array}{c}\lambda_{l} \\
(\mathrm{~nm})\end{array}$ & $\begin{array}{c}A_{l} \\
\left(\mathrm{~s}^{-1}\right)\end{array}$ & $\begin{array}{c}S_{l} \\
\text { (counts/ms) }\end{array}$ & $\begin{array}{c}p^{-1} \\
{\left[\text { photons } /\left(\text { counts } \mathrm{cm}^{2} \mathrm{sr}\right)\right]}\end{array}$ & $\begin{array}{c}\text { Error } \\
(\%)\end{array}$ & Ref. \\
\hline C VI & 2.85 & $2.17 \times 10^{11}$ & 1204 & 18.2 & $2.91 \times 10^{10}$ & 697 & $4.00 \times 10^{7}$ & 23 & 12 and 13 \\
\hline B V & 4.09 & $1.05 \times 10^{11}$ & 854 & 26.2 & $1.41 \times 10^{10}$ & 211 & $4.06 \times 10^{7}$ & 23 & 12 and 13 \\
\hline B IV & 5.26 & $1.08 \times 10^{11}$ & 430 & 38.1 & $5.10 \times 10^{9}$ & 47 & $7.67 \times 10^{7}$ & 23 & 14 \\
\hline O VI & 12.98 & $2.90 \times 10^{10}$ & 86 & 49.8 & $8.90 \times 10^{9}$ & 4 & $1.48 \times 10^{7}$ & 23 & 15 and 16 \\
\hline Ar VIII & 15.9 & $1.10 \times 10^{10}$ & 141 & 33.7 & $1.10 \times 10^{10}$ & 41 & $1.62 \times 10^{7}$ & 23 & 16 \\
\hline $\mathrm{N} \mathrm{V}$ & 18.6 & $1.40 \times 10^{10}$ & 56 & 71.4 & $4.30 \times 10^{9}$ & 3 & $9.12 \times 10^{6}$ & 23 & 15 and 16 \\
\hline
\end{tabular}

All presented line pairs were carefully checked for blending by neighboring lines and in case of an overlap by comparing the transition probabilities ${ }^{18}$ in conjunction with an estimate of the population densities with the program FLYCHK ${ }^{19}$ before their signal was integrated and background corrected. In order to obtain the inverse sensitivities for these emission lines the integrated background corrected lines were extrapolated to the same working voltages for the detectors. With the known calibration factor at $\lambda_{l}$, the intensity $I_{l}$ was calculated, and with Eq. (3) we obtained the intensity $I_{s}$ at $\lambda_{s}$. The power output $P_{s}$ at the entrance slit for $\lambda_{s}$ was calculated with the help of the solid angle determined by the distance of the entrance slit with respect to the light source and used with Eqs. (1) and (2) to determine $p^{-1}$. The error of this method is estimated to be $23 \%$. In addition to the error of the calibrated signal $(18 \%)$ the errors of the second line width integrated signal $(10 \%)$ and of the voltage extrapolation (10\%) have to be taken into account.

Figure 2 shows all calibrated points of spectrometer 2. The diamonds are the data points directly obtained from the hollow cathode; the squares are those obtained from branching ratios. Both methods provide consistent results in the overlapping region.

Spectrometer 1 was calibrated after the mounting at TEXTOR with branching line pairs which typically appear in TEXTOR plasmas: C VI 2.8/18.2 nm, B IV 5.2/38.1 nm, and B V 4.1/26.2 nm (see also Table III). The intensity $I$ of the voltage corrected signal $S$ of the emission line of the fusion plasma in the calibrated spectral region of HEXOS was derived via

$$
I=\frac{h c}{\lambda} p^{-1} S .
$$

By using Eq. (4) with Eq. (3) the inverse sensitivity $p_{s}^{-1}$ at the short wavelength can be calculated as

$$
p_{s}^{-1}=\frac{A_{s} S_{l}}{A_{l} S_{s}} p_{l}^{-1} .
$$

The indices $s$ and $l$ denote the short and long wavelengths, respectively. The data points for spectrometer 1 derived with Eq. (5) are shown in Fig. 3.

\section{ESTIMATE OF THE OPTICAL DEPTHS}

In order to estimate the applicability of the branching ratio technique we calculated the optical depths of the uti- lized emission lines. Considering induced emission, the optical depth $\tau$ (in SI units) for a Doppler-broadened line ${ }^{20}$ is

$$
\tau=3.52 \times 10^{-15} f_{i k} \lambda n_{i}^{l} \sqrt{\frac{m c^{2}}{k_{B} T_{e}}} L\left[1-\exp \left(-\frac{h c}{\lambda k_{B} T_{e}}\right)\right],
$$

with the absorbing oscillator strength $f_{i k}=1.5$ $\times 10^{-4}\left(g_{k} / g_{i}\right) \lambda^{2} A_{k i}$ with $g_{k}$ and $g_{i}$ being the statistical weights, ${ }^{21} n_{i}^{l}$ the density of the absorbing ions, $m$ the mass of the ion, $k_{B}$ the Boltzmann constant, and $L$ the length of the absorbing ion density line of sight in the plasma. The statistical weights were taken from Refs. 15 and 18.

For the determination of the optical depth $\tau$ we estimated the density of the absorbing ions $n_{i}^{l}$ with the program FLYCHK. ${ }^{19}$ In the case of the pinch light source (oxygen, argon, and nitrogen) we assumed a plasma density which resulted due to the compression of an ideal gas in the original cylindrical volume. With a plasma diameter compressed from $\approx 4$ to $0.5 \mathrm{~mm}$ at an initial pressure of $\approx 0.04 \mathrm{mbar}$ and initial gas temperature of $\approx 380 \mathrm{~K}$, the total ion density resulted in $n_{i} \approx 5 \times 10^{22} \mathrm{~m}^{-3}$. With the help of the signal ratios of the different appearing ionization stages in a recorded oxygen spectrum (Fig. 4) we roughly estimated the electron temperature $T_{e}$ to be between 30 and $40 \mathrm{eV}$. Since the pinch light source seems to produce an inhomogeneous plasma and we also observed emission lines from He-like nitrogen which

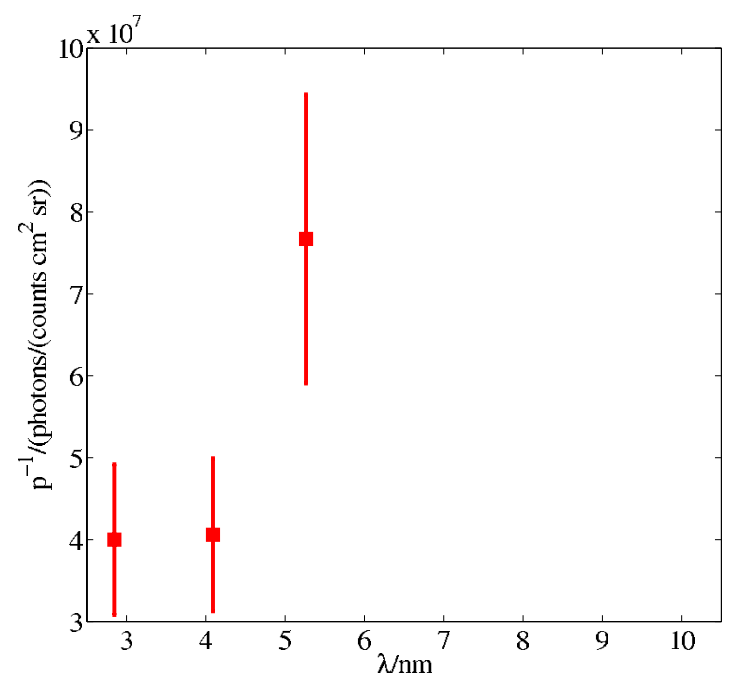

FIG. 3. (Color online) Inverse sensitivity of spectrometer 1. All data points were obtained via the branching ratio technique using TEXTOR plasmas. 


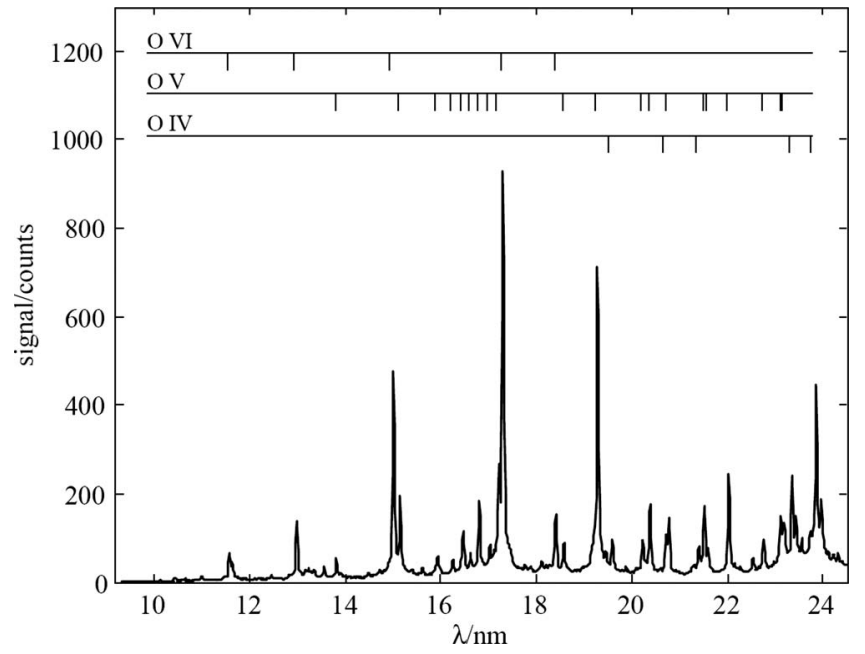

FIG. 4. Oxygen spectrum from the laboratory pinch light source recorded by HEXOS 2. This spectrum was used to roughly estimate the electron temperature.

require electron temperatures of $\approx 100 \mathrm{eV}$, a more detailed analysis of the temperature will be given in the future. As average temperature we used $T_{e}=35 \mathrm{eV}$ for the calculations. ${ }^{6}$

To estimate $n_{i}^{l}$ of carbon and boron, we estimated the impurity densities in the TEXTOR plasma with the transport code STRAHL using the wavelength ranges of HEXOS which were already calibrated with the hollow cathode (a detailed example of this procedure is given in Sec. VI). The impurity densities at the maximum of the emissivity shells of C VI, B V, and B IV derived by STRAHL are $5.5 \times 10^{17}$, $1.7 \times 10^{17}$, and $1.6 \times 10^{17}$, respectively. The electron temperatures and densities used to calculate $n_{i}^{l}$ with FLYCHK were derived by the location of the maximum of the emissivity shells.

The optical depths calculated with Eq. (6) and the parameters used for the determination are listed in Table IV. Since the optical depth for O VI $12.98 \mathrm{~nm}$ is around 0.3, the inverse sensitivity $p^{-1}$ at this wavelength is overestimated and needs special attention.

\section{APPLICATION TO TEXTOR PLASMAS}

With the help of equilibrium calculations using an impurity transport code it is now possible to give a rough estimate of impurity concentrations in the plasma. Due to the nonlinear amplification of the MCPs the calibration has to be adjusted to the actual working voltage of the spectrometer at TEXTOR. The line integrated vacuum ultraviolet intensities of prominent principal impurities are then calculated using the absolute calibration of HEXOS. For a first test we measured the line integrated radiative power of $\mathrm{H}$-like and $\mathrm{Li}$ like carbon (C VI $3.38 \mathrm{~nm}$ and C IV $38.4 \mathrm{~nm}$ ) with HEXOS and compared it to predictions by equilibrium calculations with carbon of the transport code STRAHL (Ref. 22) assuming a radial distribution of the diffusion coefficient $D(r)$ from $4 \mathrm{~m}^{2} / \mathrm{s}$ at the plasma edge to $0.1 \mathrm{~m}^{2} / \mathrm{s}$ in the plasma core and for the drift $v(r)$ of $-2 \mathrm{~m} / \mathrm{s}$ (a negative sign means an inwardly directed velocity) at the edge rising to $0 \mathrm{~m} / \mathrm{s}$ in the core. The transport coefficients and the plasma parameters
TABLE IV. Parameters used for the determination of the optical depths $\tau$. In the cases of carbon and boron, the width of the absorbing density, $L$, is the FWHM of the emissivity shell in the plasma. The electron temperatures $T_{e}$ are determined by the maximum of the emissivity shell and the absorbing ion density was calculated with FLYCHK. For oxygen, argon, and nitrogen, $L$ is the length of the pinch plasma.

\begin{tabular}{lcccccc}
\hline \hline Ion & $\begin{array}{c}\lambda \\
(\mathrm{nm})\end{array}$ & $\begin{array}{c}n_{e} \\
\left(\mathrm{~m}^{-3}\right)\end{array}$ & $\begin{array}{c}T_{e} \\
(\mathrm{eV})\end{array}$ & $\begin{array}{c}n_{i}^{l} \\
\left(\mathrm{~m}^{-3}\right)\end{array}$ & $\begin{array}{c}L \\
(\mathrm{~cm})\end{array}$ & $\tau$ \\
\hline C VI & 2.85 & $2.6 \times 10^{19}$ & 392 & $5.3 \times 10^{15}$ & 35 & $5.3 \times 10^{-6}$ \\
C VI & 18.2 & $2.6 \times 10^{19}$ & 392 & $2.0 \times 10^{7}$ & 35 & $5.4 \times 10^{-14}$ \\
B V & 4.09 & $1.7 \times 10^{19}$ & 259 & $8.7 \times 10^{14}$ & 35 & $1.6 \times 10^{-6}$ \\
B V & 26.2 & $1.7 \times 10^{19}$ & 259 & $7.5 \times 10^{6}$ & 35 & $8.8 \times 10^{-14}$ \\
B IV & 5.26 & $0.8 \times 10^{19}$ & 120 & $2.9 \times 10^{13}$ & 25 & $2.0 \times 10^{-7}$ \\
B IV & 38.10 & $0.8 \times 10^{19}$ & 120 & $4.1 \times 10^{11}$ & 25 & $1.0 \times 10^{-8}$ \\
O VI & 12.98 & $3.0 \times 10^{23}$ & 35 & $4.5 \times 10^{20}$ & 0.6 & $2.6 \times 10^{-1}$ \\
O VI & 49.8 & $3.0 \times 10^{23}$ & 35 & $2.3 \times 10^{18}$ & 0.6 & $1.3 \times 10^{-2}$ \\
Ar VIII & 15.9 & $4.0 \times 10^{23}$ & 35 & $6.5 \times 10^{18}$ & 0.6 & $5.3 \times 10^{-3}$ \\
Ar VIII & 33.7 & $4.0 \times 10^{23}$ & 35 & $4.5 \times 10^{18}$ & 0.6 & $8.5 \times 10^{-3}$ \\
N V & 18.6 & $2.5 \times 10^{23}$ & 35 & $4.8 \times 10^{19}$ & 0.6 & $3.4 \times 10^{-2}$ \\
N V & 71.4 & $2.5 \times 10^{23}$ & 35 & $5.9 \times 10^{17}$ & 0.6 & $3.4 \times 10^{-3}$ \\
\hline \hline
\end{tabular}

determine the emissivity shell of the impurities in the fusion plasma. With the help of a constant carbon influx, the predicted line intensity was adjusted to the experimental one so we derived the local carbon density in the region of the emissivity shell for this wavelength.

The peak electron density in TEXTOR pulse 102501 with neutral beam injection heating was $4.5 \times 10^{19} \mathrm{~m}^{-3}$ with a peak electron temperature of $1.2 \mathrm{keV}$. The emissivity shells of the C VI $3.38 \mathrm{~nm}$ line and C IV $38.4 \mathrm{~nm}$ line are located in the range [full width at half maximum (FWHM)] of 18-35 $\mathrm{cm}$ of the minor radius. The intensities of the C VI $3.38 \mathrm{~nm}$ line and the C IV $38.4 \mathrm{~nm}$ line measured with the calibrated HEXOS were around 5 and $0.6 \mathrm{~kW} / \mathrm{m}^{2}$, respectively, with an error of $31 \%$ (accuracy of the branching ratio calibration, $23 \%$; error of the line integrated signal, $10 \%$; voltage extrapolation, $10 \%$; correction factor for the saturation of the detector, $15 \%)$. The average carbon density derived with the equilibrium calculation of STRAHL was $5.5 \times 10^{17} \mathrm{~m}^{-3}$ in the emissivity shell with an error of about $38 \%$ (experimental intensity, 31\%; atomic data, 15\%; transport coefficients, $15 \%$; experimental input data, $5 \%$ ). This corresponds to a local carbon concentration of $2 \%$, which is equivalent to a local $Z_{\text {eff }}$ of about 1.55 , which is in the usual range of tokamaks with a carbon wall.

\section{CONCLUSION}

We provided all available calibration curves for the HEXOS system for an MCP voltage of $1150 \mathrm{~V}$ and a phosphor screen voltage of $7500 \mathrm{~V}$. The method of calibration was discussed and it was shown that the intensity calibration with a calibrated hollow cathode as secondary standard was successful. It was also shown that the branching ratio technique in combination with a pinch light source or a fusion plasma extends the intensity calibration to spectral regions which are not accessible with the hollow cathode light source. The measured data points of the hollow cathode were compared to calculated sensitivity curves to validate the cali- 
bration. Within the error bars the results are in quite good agreement with the calculations. We discussed the applicability of the branching ratio technique by estimating the optical depths of the emission lines. The TEXTOR plasma is optically thin for all used emission lines. In the case of the pinch light source the O VI $2.85 \mathrm{~nm}$ emission line shows an optical depth of around 0.3 so the inverse sensitivity at this wavelength is overestimated. Due to the absolute calibration of the HEXOS system and in conjunction with equilibrium calculations of the transport code STRAHL the concentrations of impurity ions in fusion plasmas with equilibrated transport conditions can be estimated. Measurements with carbon line radiation resulted in a local carbon density of 5.5 $\times 10^{17} \mathrm{~m}^{-3}$ around a minor radius of $18-35 \mathrm{~cm}$ in TEXTOR, which corresponds to a local carbon concentration of $2 \%$.

${ }^{1}$ W. Biel, G. Bertschinger, R. Burhenn, R. König, and E. Jourdain, Rev. Sci. Instrum. 75, 3268 (2004).

${ }^{2}$ B. Stratton, R. J. Fonck, K. Ida, K. P. Jaehnig, and A. T. Ramsey, Rev. Sci. Instrum. 57, 2043 (1986).

${ }^{3}$ N. K. Bibinov, D. O. Bolshukhin, D. B. Kokh, A. M. Pravilov, I. P. Vinogradov, and K. Wiesemann, Meas. Sci. Technol. 8, 773 (1997).

${ }^{4}$ J. Hollandt, M. Kühne, and B. Wende, Appl. Opt. 33, 68 (1994).

${ }^{5}$ J. Hollandt, M. Kühne, M. C. E. Huber, and B. Wende, Astron. Astrophys. Suppl. Ser. 115, 561 (1996).
${ }^{6}$ K. Bergmann, G. Schriever, O. Rosier, M. Müller, W. Neff, and R. Lebert, Appl. Opt. 38, 5413 (1999).

${ }^{7}$ W. Biel, G. Bertschinger, and TEXTOR Team, Rev. Sci. Instrum. 75, 2471 (2004).

${ }^{8}$ W. Biel, A. Greiche, R. Burhenn, E. Jourdain, and D. Lepere, Rev. Sci. Instrum. 77, 10F305 (2006).

${ }^{9}$ K. Danzmann, M. Günther, J. Fischer, M. Kock, and M. Kühne, Appl. Opt. 27, 4947 (1988).

${ }^{10}$ A. S. Tremsin and O. H. W. Siegmund, Proc. SPIE 3765, 441 (1999).

${ }^{11}$ D. G. Simons, G. W. Fraser, P. A. J. De Korte, J. F. Pearson, and L. De Jong, Nucl. Instrum. Methods Phys. Res. A 261, 579 (1987).

${ }^{12}$ J. Z. Klose and W. L. Wiese, J. Quant. Spectrosc. Radiat. Transf. 42, 337 (1989).

${ }^{13}$ E. Hinnov and F. W. Hofmann, J. Opt. Soc. Am. 53, 1259 (1963).

${ }^{14}$ R. Lincke, Plasma Diagnostics (North-Holland, Amsterdam, 1968).

${ }^{15}$ D. A. Verner, E. M. Verner, and G. J. Ferland, At. Data Nucl. Data Tables 64, 1 (1996).

${ }^{16}$ V. G. Pal'chikov, Phys. Scr. 57, 581 (1998).

${ }^{17}$ A. W. Weiss, NIST Atomic Spectra Database, Version 3.1.2, 1964.

${ }^{18}$ NIST Atomic Spectra Database, 2007 (http://physics.nist.gov/ PhysRefData/ASD/index.html).

${ }^{19}$ H.-K. Chung, M. H. Chen, W. L. Morgan, Y. Ralchenko, and R. W. Lee, High Energy Density Phys. 1, 3 (2005).

${ }^{20}$ J.-D. Huba, NRL Plasma Formulary (Naval Research Laboratory, Washington, DC, 2005).

${ }^{21}$ J. Richter, Plasma Diagnostics (North-Holland, Amsterdam, 1968).

${ }^{22}$ R. Dux, STRAHL User Manual (Max-Planck-Institut für Plasmaphysik, Garching, 2006). 